\title{
Omnidirectional Multi-scale Generalized Blur Minimization Mathematical Morphology Edge Detection Algorithm
}

\author{
${ }^{1}$ Yang Shubin, ${ }^{2}$ Qiu Qianwen, ${ }^{3}$ Zhang Sai, ${ }^{4}$ LI Jinpeng \\ School of Electrical and Information Eng., Wuhan Institute of Technology \\ Wuhan, 430073, P.R. China \\ 1yshubin@sina.com, ²qqwen@126.com, ${ }^{3}$ zhangsai@sina.com, ${ }^{4}$ jinpengli@163.com
}

\begin{abstract}
Owing to image noise and differ in thousands ways of edge shape, traditional morphological edge detection operators can't detect the edge well and the edge detected by it is very blur. In order to gain the good edge, characteristic of morphological operators are used. Generalized morphological detection operator is used to conquer noise influence, Omnidirectional edge operator is used to detect different direction edge information and multi-scale edge operator is used to gain different scale and detail edge information and wipe off noise more. After that, according to suitable weights combining edge result to make up another edge image which can gain final edge through blur-minimization edge detection. Experiment proved that the proposed edge detection algorithm can detect edge better than traditional detection algorithm and can not only detect image edge effectively with upper detection precision but also restrain noise efficaciously.
\end{abstract}

Keywords - Mathematical Morphology; Edge Detection; Omnidirectional Mulit-scale; Blur Minimization

\section{INTRODUCTION}

Image edge is the mutation region of the gray level or structure position and also the dividing line between target and background. It has invariability and can draw the basic outline of target object. So it is one of basic image feature. Edge detection has wondrously consequence in image processing and computer vision. Image edge ascertained is an indispensable prerequisite in image analysis and recognition. It is contributed to the whole image scene recognition and understanding. But edge detection accuracy will directly affect the subsequent image processing. It is one of important classical research project in image segmentation field.

Common edge detection algorithm is fulfilled through computing the derivative of image gray function. That is, through extreme value of First order derivative or zero-crossing information of two order derivative to judge points of the edge[1]. These algorithms often are the same with some specific type images. For example, it can only be suitable for images with less noise and less complex shape. So it is difficult to content demand of complex images. At present, many burgeoning subject theory and concept were imported and combined with each other for edge detection. Such as Chen Wufan introduced concept of generalized blur set[2]and implemented edge detection through selecting appropriate subjection functions to construct generalized blur operator; Zhang Baohua[3] proposed multi-scale edge detection algorithms based on orthogonal wavelet transform to detect edge from different resolution using characteristic of multi-scale wavelet transform to restrain noise; $\mathrm{Li}$ Cuihua[4] marks relaxation gridding pixels obtained by edge information and detects edge using underrelaxation iterative method. And theory of mathematical morphology is also applied in edge detection. With the development and perfection of mathematical morphology research, its operation has natural characteristics to establish difference operator. The extraction edge is smooth and retain image details features. It has good anti-noise performance and can preferably reserve image details. At the same time it can satisfy the real-time requirement. Combining the advantages of all kinds of morphological algorithm in edge detection, omnidirectional mulit-scale generalized blur minimization mathematical morphology edge detection algorithm is designed to detect image edge and It has a good effect.

\section{II.BRIEF INTRODUCTION OF MORPHOLOGICAL EDGE}

\section{DETECTION ALGORITHM}

Basic morphological gradient BMG[5,6] can be constructed by definitions and properties of basic operations. The edge obtained by erosion type edge operator is the Inner 
edge of image. The edge obtained by dilation type edge operator is the outside edge of image. And the edge obtained by erosion-dilation type edge operator is the summation of Inner edge and the outside edge. In fact, it is the image edge across the edge of euclidean boundary. Although the three edge operators are sensitive to noise, they do not strengthen or enlarge noise and bring out phenomenon of false positive edge. Opening type edge operator can be used to detect the peak value of the image. Closing edge operator is the dual operator of opening type operator and can detect the image valley value. Opening-closing type operator can detect the image peak value and the valley value at the same time. But it will produce big deviation. So the anti-noise performance of these three operators are better than the former three operators. But image offset still exists in the obtained edge results. In order to improve detection effect, generalized morphological edge detection[7] and omni-direction mulit-scale edge detection algorithms[8] are proposed. Integrated operator can decrease phenomenon of blur while the detected image contains more noise. The operators smooth image at first and then detect edge. Due to its decreasing blur, it is called Blur-Minimization Edge Detection blur minimization morphological edge detection and also called BM operator[6] . It can inhibit the noise very well and be suitable for ideal edge detection. But it is not ideal to the step edge And it is hard to eliminate isolated noise points. As a kind of nonlinear difference operator, In fact, the basic morphological edge detection algorithm is a certain extent extension of difference operator. And also in the several edge detection operators in above, selecting structural element is a key step. The edge detection results are directly affected by structural elements.

\section{ALGORITHM DESIGN AND ANALYSIS}

Applying theory of generalized morphological detection algorithm and omnidirectional mulit-scale structure element[8,9], combining with BM operator, Omnidirectional mulit-scale generalized blur minimization mathematical morphology edge detection algorithm is proposed. Detail steps are designed as follows:

(1) Choosing basic structural element $B$, after $(n-1)$ times dilation iterative computation, multi-scale structural elements $n B$ with scale parameter $n$ are obtained. Although anti-noise performance of large scale structural element is better, while the element scale increases to a certain extent, improvement of anti-noise performance will not change a lot. Instead, It leads to increase edge thickness and make image edge details blur. Therefore, maximum of $n$ often sets as 4 6 times.

(2) For multi-scale structural element $n B$ with scale parameter $\mathrm{n}$, constructing $W_{n}$ multi-scale structural elements.

$$
\text { Where } \quad W_{n}=\left\{\begin{array}{cc}
2 & n=1 \\
4(n-1) & n \geq 2
\end{array}\right.
$$

Sub structural element of the $i$ th direction in $W_{n}$ multi-scale structural element $n B$ was $n B_{i}$;

(3) Detect the $i$ th direction edge $n G_{1}(i)$ of the direction $n B_{i}$ with sub structural element of the $i$ th direction in $W_{n}$ multi-scale structural element $n B$

$$
n G_{1}(i)=\left[\left(f \oplus B_{1}\right) \Theta B_{2}\right] \circ n B_{i}-\left[\left(f \oplus B_{1}\right) \Theta B_{2}\right] \Theta n B_{i}
$$

Then edge $n G_{1}$ of multi-scale structural element $n B$ can be obtained through weight adding edges gotten from each direction. That is

$$
n G_{1}=\sum_{i=0}^{W_{n}-1} \phi_{i} * n G_{1}(i)
$$

Where $\phi_{i}$ is the weight of the ith direction edge in the multi-scale structural element $n B$. Since omnidirectional sub structural elements $n B_{i}$ of the multi-scale structural element $n B$ have the same scale parameter $n$, it can be considered that contribution of the edge extracted from each direction is same for edge synthesis. So the weights are all 
set as $\frac{1}{W_{n}}$, then $n G_{1}=\sum_{i=0}^{W_{n}-1} \frac{1}{W_{n}} * n G_{1}(i)$

(4) Get edge detection information $\operatorname{Grad}_{1}$ of erosion operator based on multi-scale omnidirectional structural elements through syncretizing edges of multi-scale structural element $n B$ in different scales. It is

$$
\operatorname{Grad}_{1}=\sum_{n=k}^{l} \omega_{n} * n G_{1}
$$

Thereinto $\omega_{n}$ is the edge weight of structural elements $n B$ in different scales. $[k, l]$ is the value range of scale parameter $n$. The weight $\omega_{n}$ can computed by the adaptive method ${ }^{[8]}$.

(5) Edge information $\mathrm{Grad}_{2}$ of dilation operator and $\mathrm{Grad}_{3}$ of dilation-erosion operator based on multi-scale omnidirectional structural elements are obtained by repeating process (3) and (4) through dilation and dilation-erosion type detection operator . They are:

$$
\begin{aligned}
& \operatorname{Grad}_{2}=\sum_{n=k}^{l} \omega_{n} *\left[\sum_{i=0}^{W_{n}-1} \frac{1}{W_{n}} *\left(f \Theta B_{1} \oplus B_{2} \oplus n B_{i}-f \Theta B_{1} \oplus B_{2} \bullet n B_{i}\right)\right] \\
& \operatorname{Grad}_{3}=\sum_{n=k}^{l} \omega_{n} *\left[\sum_{i=0}^{W_{n}-1} \frac{1}{W_{n}} *\left(f \Theta B_{1} \oplus B_{2} \oplus n B_{i}-f \oplus B_{1} \Theta B_{2} \Theta n B_{i}\right)\right]
\end{aligned}
$$

(6)Edge detection result $\mathrm{Grad}_{2}$ based on erosion operator may drop some image edge details and edge detection result $\mathrm{Grad}_{3}$ based on dilation operator often makes edge blur. In order to avoid these disadvantage the above blur minimization morphological edge detection algorithm (BM operator ) can be inducted. It can reduce noise blur at first, then edge is detected by mathematical morphological edge detection algorithm. On the basis of theory about edge information $\operatorname{Grad}_{1}, \mathrm{Grad}_{2}$ and
Grad $_{3}$ obtained former are composited using blur minimization BM operator. The composited results can gained details information which can be used to reduce edge blur. Obvious perfect edge GRAD is gained by inosculating edge information into edge $\mathrm{Grad}_{3}$. Computing method is shown as formula (6),(7),(8)and (9).

$$
\begin{aligned}
G_{\text {max }} & =\max \left\{\operatorname{Grad}_{1}, \operatorname{Grad}_{2}, \operatorname{Grad}_{3}\right\} \\
G_{\text {min }} & =\min \left\{\operatorname{Grad}_{1}, \operatorname{Grad}_{2}, \operatorname{Grad}_{3}\right\} \\
G_{\text {mn }} & =G_{\text {max }}-G_{\text {min }}
\end{aligned}
$$

$$
G R A D=\operatorname{Grad}_{3}+0.5 * G_{m n}
$$

\section{EXPERIMENTS AND ANALYSIS}

The proposed algorithm is used on brain MRI image to analyze and verify its validity. Classical edge detection operators are used operator Prewitt and LOG. $3 \times 3$ crisscross structural element and anti-noise dilation-erosion operator are chosen as single structure element and edge detection operator. The value, range of the element scale parameter in the proposed algorithm in this paper is [1,4]. The basic structural element choose $3 \times 3$ crisscross element. The experiment results are shown in Figure 1. It is obvious from visual analysis of result image that Prewitt operator can only give a vague description of the outline of brain and the edge obtained is incoherent. Also the edge positioning precision is not high; Although LOG operator can detect the lateral contour of brain clearly and edge details effectively such as the pituitary gland and the insular cortex, there are some breakpoints in edge image and it is difficult to detect some region whose contrast is low. The ability of single structure element anti-noise edge detection algorithm is close to LOG operator's, but its edge continuity is better. Although the gray scale of every part in brain MRI image are close, the edge detail information can be gained clearly using the proposed algorithm. In order to evaluate detection effect, connected component ratio[11] and signal-to-noise ratio are used to quantitative evaluation. Connection component can 
be used to estimate edge effect through measuring linear

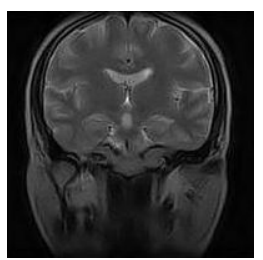

(a)

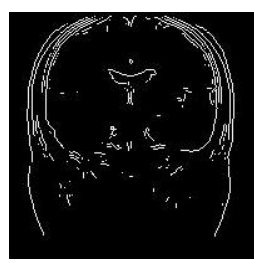

(b)

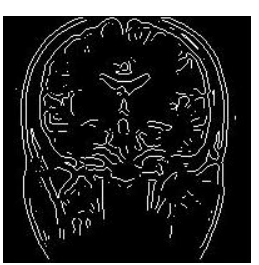

(c)

connection degree by the ratio $\mathrm{A} / \mathrm{B}$ between number $\mathrm{A}$ of

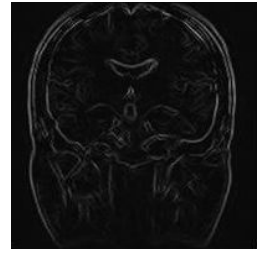

(d)

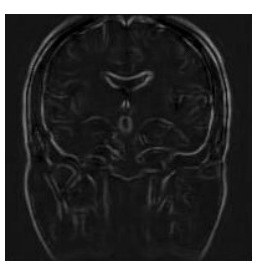

(e)

Figure 1 Edge detection of the MRI image of the brain

(a)original MRI image (b) Prewitt operator (c) LOG operator (d) anti-noise dilation erosion operator (e)the proposed algorithm

4-connected components and $\mathrm{B}$ of 8-connected components. It can be known that the smaller ratio $\mathrm{A} / \mathrm{B}$, the less possibility of leak or wrong detection, the better detection result. The signal-to-noise ratio can measure anti-noise performance. Experimental data are shown in table.1. It can be seen from table 1 that connected component ratio of the propose algorithm is the least. It means that the degree of edge line connection is the highest and the less possibility of leak or wrong detection is the least, so detection effect is the best. Simulttaneity, signal-to-noise ratio of the proposed algorithm is the highest. That means that its anti-noise performance is the best in the several algorithm. From the results of experimental image and the data analysis, It can drawn that the proposed algorithm can restrain the noise well and the edge detection effect is satisfied. The edge obtained not only has the clear details but also has good consistency and is better than other edge detection algorithm.

TABLE 1. CONNECTED COMPONENT RATIO AND SIGNAL-TO-NOISE RATIO

\begin{tabular}{c|c|c}
\hline algorithm & $\begin{array}{c}\text { connected } \\
\text { component ratio }\end{array}$ & $\begin{array}{c}\text { signal-to-noise ratio } \\
\text { (dB) }\end{array}$ \\
\hline Prewitt operator & 0.5322 & 12.15 \\
\hline LOG operator & 0.3696 & 13.42 \\
\hline $\begin{array}{c}\text { anti-noise dilation } \\
\text { erosion operator }\end{array}$ & 0.1264 & 14.85 \\
\hline $\begin{array}{c}\text { the proposed algorithm } \\
\text { in this paper }\end{array}$ & 0.08923 & 15.06 \\
\hline
\end{tabular}

\section{CONCLUSION}

As important part of image processing and analysis, edge detection has been widely studied and applied for a long time. On the basis of nonlinear mathematical morphology, omnidirectional multi-scale generalized blur minimization mathematical morphology edge detection algorithm is used to detect edge information by constructing mulit-scale and omnidirectional structure element, generalized anti-noise edge detection method, then choosing suitable weights to synthesize edge and modifying it by reducing the blur degree to gain final edge. Experiment proved that the proposed algorithm can detect edge better and not only detect image edge effectively with upper detection precision but also restrain the noise efficaciously.

\section{REFERENCES}

[1] Zhang Yujin. Image Processing and Analysis[M]. Bejing : Higher Education Press, 2002.

[2] Chen Wufan. A New Algorithm of Color Images Edge Detection-GFO Algorithm[J].China Science(A), 1995, 25(2): $219-220$.

[3] Zhang Baohua,Ye Hua,Chen Weinan. A Multiscale Edge Detector Based on Orthonormal Wavelets Transform[J]. Journal of Image and Graphics, 1998, 8(3) : 12-14.

[4] Li Cuihua,Zheng Nanning,Zhang Yongping. An Underrelaxation Scheme for a Fast Adaptive Filter[J]. Journal of Xi'an Jiaotong University, 1998, 32(11) : 1-4.

[5] Wang Yongsheng. Research of Edge Deteetion for Grey - Scale Image Based on Mathematical Morphology[D]. Harbin,The thesis for Master Degree of Harbin Engineering University, 2005.

[6] James Lee.J. Morphological Edge Detection[J]. IEEE Journal of Robotics and Automation, 1987, RA-3(2) : 142-156.

[7] Yang Shubin,Peng Fuyuan. Application of Morphological Edge Detectors in Image Corrupted by Noise[J].Computer Engineering and Applications, 2002, 38(17) : 91-92.

[8] Yang Shubin,Peng Fuyuan,Zhang Zhengchang. Morphological Edge Detector with Multiscale Adaptive Weighting[J].Hua Zhong University of science and Technology, 2002, 30(10) : 107-111.

[9] Wang Liangliang,Li Ming,Gao Xin. Edge Detection Algorithm of Heavy Noisy Image Based on Multi- Direction and Multi-Scale. Journal of Spacecraft TT \& C Technology, 2010, 29 (4) : 33-36.

[10] Chen Yanyan,Wang Yuanqing. Quantitative Comparison of Common Edge Detection Algorithms[J]. Computer Engineering, 2008, 17(34) : 202-204. 